\title{
Erosividade da chuva para o bioma Pantanal
}

\author{
Rainfall erosivity for Pantanal biome \\ Daniel Okamoto Machado', Teodorico Alves Sobrinho², Aline da Silva Ribeiro³, \\ Carlos Nobuyoshi Ide ${ }^{4}$, Paulo Tarso Sanches Oliveira ${ }^{5}$
}

\begin{abstract}
口-
\section{RESUMO}

A escassez de dados pluviográficos na região do Pantanal fez com que muitos autores utilizassem equações de regressão obtidas em outras regiões do Brasil para calcular o Fator R da Equação Universal de Perda de Solo (USLE). O presente estudo define equações para a previsão da erosividade em região do bioma Pantanal, correlacionando a erosividade das chuvas calculada pelo índice $\mathrm{EI}_{30}$ e o coeficiente de chuva (Rc). A equação desenvolvida, do tipo potencial, apresenta consistência e correlação significativa com os dados observados, obtendo valor de coeficiente de determinação de 97\%. Portanto, a equação de erosividade proposta pode ser utilizada para estimativa do fator R da USLE a partir dos dados mensais e anuais de precipitação da região e de outras regiões com características climáticas semelhantes.
\end{abstract}

Palavras-chave: perda de solo; erosividade; conservação do solo; erosão,

\begin{abstract}
The scarcity of pluviograph data in the Pantanal zone forced many researchers to use regression equations obtained in others regions of Brazil to calculate the R factor of Universal Soil Loss Equation (USLE). This study defines a model to predict the erosivity of the Pantanal Biome correlating the calculated rainfall erosivity index $\mathrm{El}_{30}$ and the rainfall coefficient (Rc). The model showed a significant correlation with the observed data, yielding coefficient of determination of $97 \%$. Therefore, the rainfall erosivity model proposed can be used to estimate the R Factor of USLE data from monthly and annual precipitation for the region and other regions with similar climatic characteristics.
\end{abstract}

Keywords: soil loss; erosivity; soil conservation; erosion.

\section{INTRODUÇÃO}

As mudanças climáticas podem proporcionar alterações nas características da chuva, constituindo, assim, preocupação para a conservação do solo (MEUSBURGER et al., 2012). Segundo Collinschonn, Tucci e Clarke (2001), a região da bacia do Rio Paraguai, a qual abrange áreas dos Estados de Mato Grosso, Mato Grosso do Sul e três países vizinhos: Argentina, Paraguai e Bolívia, sofreu flutuações climáticas nos últimos tempos, com modificações dos padrões de precipitação.

A Bacia do Alto Paraguai (BAP), com área de $140.000 \mathrm{~km}^{2}$ de planície de inundação, pela sua característica e localização, destaca-se como importante área do Pantanal Sul-mato-grossense (HARRIS et al., 2005; PAZ et al., 2011). A BAP é definida pela seção em que o Rio Paraguai recebe a contribuição do Rio Apa, na fronteira entre o Brasil e o Paraguai. O Pantanal caracteriza-se por ser de região baixa, localizada no centro da bacia, onde os rios inundam a planície e alimentam um intrincado sistema de drenagem que inclui lagos extensos, cursos d'água divergentes e áreas de escoamento e inundação sazonal (REBOUÇAS; BRAGA; TUNDISI, 2002).

Este sistema vem sendo ameaçado pelas agroindústrias e reservatórios de hidrelétricas que modificam os padrões de vazões e a carga de sedimentos dos rios (JUNK \& CUNHA, 2005). As atividades agropecuárias na área vêm aumentando ainda o suprimento de sedimento do Pantanal, transformando a erosão do solo em problema ambiental nessa bacia (ASSINE, 2005). Segundo Salles e Poesen (2000), a perda de solo por erosão intersulcos está intimamente ligada às propriedades da precipitação, devido à energia do impacto das gotas de chuva na superfície do solo e pela sua contribuição para o escoamento superficial. Dentre os fatores que regulam a erosão do solo, a erosividade da chuva

\footnotetext{
$\square$

'Mestre em Saneamento Ambiental e Recursos Hídricos pela Universidade Federal de Mato Grosso do Sul (UFMS) - Campo Grande (MS), Brasil.

2Doutor em Engenharia Agrícola pela Universidade Federal de Viçosa (UFV). Professor associado da UFMS. Bolsista do CNPq - Viçosa (MG), Brasil.

${ }^{3}$ Engenheira Ambiental pela UFMS. Mestre em Hidráulica e Saneamento pela Escola de Engenharia de São Carlos da Universidade de São Paulo (EESC/USP). Doutoranda em

Engenharia Hidráulica Sanitária pela Escola Politécnica da Universidade de São Paulo (POLI/USP). Pesquisadora do Instituto de Pesquisas Tecnológicas (IPT/SP) - São Paulo (SP), Brasil. ${ }^{4}$ Engenheiro Civil pela UFMS. Mestre e Doutor em Recursos Hídricos e Saneamento Ambiental pela Universidade Federal do Rio Grande do Sul (UFRGS). Professor associado da UFMS Campo Grande (MS), Brasil.

${ }^{5}$ Doutorando em Hidráulica e Saneamento pela EESC/USP - São Carlos (SP), Brasil.

Endereço para correspondência: Daniel Okamoto Machado - Faculdade de Engenharias, Arquitetura e Urbanismo e Geografia da Universidade Federal de Mato Grosso do Sul Cidade Universitária, s/n - Caixa Postal 549 - 79070-900 - Campo Grande (MS), Brasil - E-mail: okamachado@gmail.com

Recebido: 18/07/12 - Aceito: 07/01/14 - Reg. ABES: 474
} 
tem maior importância por ser difícil de predizer e de ser controlada pelo homem (ANGULO-MARTÍNEZ \& BEGUERÍA, 2009). A erosividade da chuva expressa a sua capacidade de causar erosão em área sem proteção, sendo representada pelo Fator R da Equação Universal da Perda de Solo (USLE) (SILVA; WIECHETECK; ZUERCHER, 2011).

Wischmeier (1959) definiu a erosividade como "o produto da energia cinética de determinada chuva $\left(\mathrm{E}_{\mathrm{c}}\right)$ pela sua máxima intensidade em 30 minutos $\left(\mathrm{I}_{30}\right)$ ". Entretanto, a utilização dessa metodologia depende do conhecimento da distribuição horária ou sub-horária da intensidade de chuva. Dados de intensidade de chuva são obtidos por pluviógrafos ou por pluviômetros com datalogger. Porém, registros desse tipo ainda são escassos em muitas partes do mundo, especialmente nos países em desenvolvimento (YU; HASHIM; EUSOF, 2001). Portanto, a capacidade de se estimar a erosividade através de dados mensais e anuais de precipitação é fundamental, considerando a disponibilidade desses registros para longos períodos.

O coeficiente de chuva (Rc), modificado por Lombardi Neto (1977), expressa a relação entre o quadrado da precipitação média mensal $(p)$ e a média anual total $(P)$, para cada mês do ano, sendo esses dados comumente registrados em estações meteorológicas. Apesar da irregularidade temporal da chuva, considerada por Mikos, Jost e Petkovsek (2006) como obstáculo para a utilização desse tipo de metodologia, o Rc demonstra satisfatória relação com a erosividade da chuva (CASSOL et al., 2008).

Alguns pesquisadores (SORIANO; GALDINO; CLARKE, 2001; GALDINO; PADOVANI; SORIANO, 2004; SILVA, 2004) utilizam as equações desenvolvidas para Campinas por Lombardi Neto (1977), modificadas por Lombardi Neto e Moldenhauer (1992), apesar de essa localidade ter características distintas das observadas para a região da BAP. Isso ocorre pela escassez ou mesmo inexistência de série de dados oficiais de intensidade de chuva nessa região do Pantanal.

Assim, o presente estudo teve por objetivo desenvolver e calibrar equações para estimativa da erosividade da chuva para a BAP, correlacionando a erosividade das chuvas calculada pelo índice $\mathrm{EI}_{30}$ e o coeficiente de chuva $(\mathrm{Rc})$.

\section{METODOLOGIA}

A área de estudo compreende a bacia hidrográfica do Rio Taquarizinho, sub-bacia do Rio Taquari, uma das áreas mais impactadas do bioma Pantanal. A bacia do Rio Taquari é, por sua vez, a maior sub-bacia da BAP, localizada no Pantanal Sul-mato-grossense. A bacia do Rio Taquarizinho está localizada entre as latitudes $18^{\circ}$ e $20^{\circ} \mathrm{S}$ e as longitudes $54^{\circ}$ e $55^{\circ} 30^{\prime}$ W. A área está posicionada entre as cidades de Coxim e São Gabriel do Oeste, Mato Grosso do Sul, região dos planaltos com cotas acima de 200 $\mathrm{m}$. A região possui, predominantemente, atividade agropecuária. Em geral, se cultiva soja e milho no verão, com entressafras de milheto e sorgo para cobertura do solo, e pecuária extensiva de corte como atividade principal
(IBGE, 2010). Foram utilizados registros pluviométricos de uma série de quatro anos devido ao escasso histórico de informações climáticas no Brasil, como justificado por Oliveira et al. (2011). Os dados foram obtidos através de três pluviógrafos com datalogger instalados na bacia, tendo como fonte resultados de pesquisa do grupo iniciado no local em 2005.

A erosividade das chuvas foi determinada pelo índice $\mathrm{EI}_{30}$ para cada chuva individual erosiva, de acordo com critérios de chuva individual utilizados por Cassol et al. (2008). A energia cinética unitária, transformada por Foster et al. (1981) para o sistema internacional e apresentada em Lombardi Neto e Mondehauer (1992), foi determinada para cada segmento uniforme de chuva (Equações 1 e 2).

$e=0,119+0,0873 \log _{10} i$

para $i \leq 76 \mathrm{~mm} \cdot \mathrm{h}^{-1}$

$e=0,283 i$

para $i>76 \mathrm{~mm} \cdot \mathrm{h}^{-1}$

Onde,

$e$ : energia cinética unitária $\left(\mathrm{MJ} \cdot \mathrm{ha}^{-1} \cdot \mathrm{mm}^{-1}\right)$;

$i$ intensidade da chuva $\left(\mathrm{mm} \cdot \mathrm{h}^{-1}\right)$.

A energia cinética do segmento, em MJ.ha-1 ${ }^{-1}$ corresponde ao produto entre a energia cinética unitária e a quantidade de chuva, no respectivo segmento uniforme. A energia cinética total da chuva $\left(\mathrm{E}_{\mathrm{c}}\right)$ é obtida pela soma da energia cinética de cada segmento uniforme. A erosividade da chuva é calculada pela Equação 3.

$E I_{30}=E_{c} \cdot I_{30}$

Onde,

$E I_{30}$ : índice de erosividade da chuva erosiva individual $\left(\mathrm{MJ} \mathrm{mm} \mathrm{ha} \mathrm{m}^{-1} \mathrm{~h}^{-1}\right)$; $E_{c}$ : energia cinética total da chuva em $\left(\mathrm{MJ} \cdot \mathrm{ha}^{-1}\right)$;

$I_{30}$ : intensidade máxima da chuva em período contínuo de $30 \mathrm{~min}$ de chuva $\left(\mathrm{mm} \mathrm{h}^{-1}\right)$.

A erosividade mensal das chuvas é obtida somando-se os índices $E I_{30}$ das chuvas individuais erosivas no mês e a erosividade anual se

Tabela 1 - Classificação da erosividade das chuvas pelo índice R.

\begin{tabular}{l|c} 
Erosividade (MJ mm year ${ }^{-1} \mathbf{h a}^{-1}{ }^{-1}$ ) & Classificação da erosividade \\
\hline $\mathrm{R} \leq 2452$ & Erosividade fraca \\
\hline $2452<\mathrm{R} \leq 4905$ & Erosividade moderada \\
\hline $4905<\mathrm{R} \leq 7357$ & Erosividade moderada a forte \\
\hline $7357<\mathrm{R} \leq 9810$ & Erosividade forte \\
\hline $\mathrm{R}>9810$ & Erosividade muito forte \\
\hline
\end{tabular}

Fonte: Carvalho (2008), modificado para o S.I. métrico de unidades segundo Foster et al. (1981). 
obtém pela soma dos índices mensais $E I_{30}$, índice este que representa os valores observados. Os resultados obtidos foram classificados de acordo com as classes propostas por Carvalho (2008) (Tabela 1).

Os registros pluviométricos da série de dados entre 2006 e 2009 foram utilizados para estabelecer equações a partir das regressões linear e potencial, correlacionando os valores observados e o coeficiente de chuva proposto por Lombardi Neto (1977). A calibração das equações foi realizada pelo método de otimização Simplex. Além disso, para efeito de comparação, utilizou-se a equação desenvolvida por Lombardi Neto e Moudenhauer (1992) para Campinas com a mesma série de dados, uma vez que Silva (2004) sugeriu a aplicação dessa equação para a região da BAP.

A qualidade do ajuste das equações foi avaliada pelos coeficientes: de determinação $\left(\mathrm{R}^{2}\right)$; de Nash-Sutcliffe (COE) (Equação 4); de massa residual (CMR) (Equação 5); de ajuste (CA) (Equação 6) e pela eficiência (EF) (Equação 7).

$C O E=1-\left[\left(\sum_{i=1}^{n} O_{i}-\sum_{i=1}^{n} P_{i}\right)^{2} / \sum_{i=1}^{n}\left(O_{i}-\bar{O}\right)^{2}\right]$

$C M R=\left(\sum_{i=1}^{n} O_{i}-\sum_{i=1}^{n} P_{i}\right) / \sum_{i=1}^{n} O_{i}$

$C A=\sum_{i=1}^{n}(O-\bar{O})^{2} / \sum_{i=1}^{n}(P-\bar{O})^{2}$

$E F=\left[\sum_{i=1}^{n}\left(O_{i}-\bar{O}\right)^{2} / \sum_{i=1}^{n}\left(O_{i}-\bar{P}\right)^{2}\right] / \sum_{i=1}^{n}\left(O_{i}-\bar{O}\right)^{2}$

Onde,

$\mathrm{O}_{\mathrm{i}}$ : valores observados;
$\mathrm{P}_{\mathrm{i}}$ : valores calculados;

n: número de observações;

$\bar{O}$ : média aritmética das observações;

$\bar{P}$ : média aritmética dos valores estimados.

A análise foi feita considerando os seguintes limites de valores: quanto mais próximo de zero o valor de CMR e mais próximo de 1 os valores de COE e CA, melhor o ajuste. O coeficiente de Nash-Sutcliffe prioriza a eficiência dos valores estimados mais altos e pode variar de menos infinito a 1 . A eficiência igual ao valor unitário corresponde à combinação perfeita da equação aos dados observados e eficiência zero indica que as previsões são tão precisas quanto a média dos valores observados. Eficiência negativa ocorre quando a média observada é melhor do que a equação (SCHAEFLI \& GUPTA, 2007).

\section{RESULTADOS E DISCUSSÃO}

\section{Pluviosidade}

A precipitação média anual na área estudada, no período de 2006 a 2009, foi de $1.371 \mathrm{~mm}$ (Tabela 2), com variação anual de $1.153 \mathrm{~mm}$ (2007) até $1.699 \mathrm{~mm}$ (2009). Uma série de registros com mais de 60 anos de dados pluviométricos, de região próxima à área estudada, demonstra que a pluviosidade anual na região varia entre 1.150 e $1.500 \mathrm{~mm}$ (COLLINSCHONN; TUCCI; CLARKE, 2001). Segundo Renard, Foster e Weesies (1997), poucos eventos de chuvas torrenciais são responsáveis pelas altas taxas de sedimentos, eventos estes não identificados

Tabela 2 - Valores de precipitação pluvial ( $\mathrm{mm}$ ) no Rio Taquarizinho: acumulados mensais, acumulados anuais e médios dos acumulados mensais e anuais do período de 2006 a 2009, com suas medidas estatísticas de dispersão.

\begin{tabular}{|c|c|c|c|c|c|c|c|c|c|c|c|c|c|}
\hline Ano & jan & fev & mar & $\mathrm{abr}$ & maio & jun & jul & ago & set & out & nov & dez & Total \\
\hline 2006 & - & - & - & - & 81 & 4 & 0 & 21 & 90 & 99 & 168 & 155 & - \\
\hline 2008 & 232 & 216 & 111 & 90 & 59 & 4 & 0 & 5 & 18 & 192 & 126 & 258 & 1.314 \\
\hline Média & 277 & 233 & 131 & 60 & 61 & 7 & 26 & 20 & 43 & 129 & 174 & 211 & 1.371 \\
\hline DP & 143 & 32 & 72 & 31 & 23 & 8 & 31 & 24 & 41 & 100 & 36 & 169 & - \\
\hline CV(\%) & 52 & 14 & 55 & 52 & 38 & 118 & 119 & 118 & 95 & 78 & 21 & 80 & - \\
\hline
\end{tabular}

DP: desvio-padrão; CV: coeficiente de variação; Máx: valor máximo da série; Mín: valor mínimo da série. 
Tabela 3 - Valores mensais, anuais e médios mensais do índice de erosividade EI30 (MJ.mm/ha.h) para a bacia do Rio Taquarizinho e suas medidas estatísticas de dispersão.

\begin{tabular}{|c|c|c|c|c|c|c|c|c|c|c|c|c|c|}
\hline Ano & jan & fev & mar & $\mathrm{abr}$ & maio & jun & jul & ago & set & out & nov & dez & Total \\
\hline 2006 & - & - & - & - & 314 & 0 & 0 & 17 & 271 & 257 & 1.021 & 965 & - \\
\hline 2007 & 4.590 & 2.135 & 876 & 61 & 266 & 0 & 461 & $\mathrm{O}$ & 0 & 0 & 2.586 & 2 & 10.976 \\
\hline 2008 & 934 & 1.352 & 330 & 314 & 207 & 0 & 0 & 0 & 0 & 1.403 & 984 & 2.637 & 8.159 \\
\hline 2009 & 936 & 1.950 & 2.128 & 477 & 128 & 28 & 1.035 & 129 & 219 & 1.781 & 1.679 & 3.927 & 14.418 \\
\hline Média & 2.153 & 1.812 & 1.111 & 284 & 229 & 7 & 374 & 37 & 123 & 860 & 1.568 & 1.883 & 10.440 \\
\hline DP & 2.110 & 410 & 922 & 210 & 80 & 14 & 492 & 62 & 143 & 865 & 750 & 1.744 & - \\
\hline CV(\%) & 98 & 23 & 83 & 74 & 35 & 200 & 131 & 170 & 117 & 101 & 48 & 93 & - \\
\hline Máx & 4.590 & 2.135 & 2.128 & 477 & 314 & 28 & 1.035 & 129 & 271 & 1.781 & 2.586 & 3.927 & 4.590 \\
\hline Mín & 934 & 1.352 & 330 & 61 & 128 & 0 & 0 & 0 & 0 & 0 & 984 & 2 & 0 \\
\hline
\end{tabular}

DP: desvio-padrão; CV: coeficiente de variação; Máx: valor máximo da série; Mín: valor mínimo da série.

em séries históricas pequenas. Entretanto, os dados de precipitação do presente estudo mostraram-se uma série representativa, englobando os valores mínimos e máximos de precipitação da região. Em relação às médias, os meses de dezembro, janeiro e fevereiro apresentaram maiores valores de pluviosidade média, respectivamente 211, 277 e $233 \mathrm{~mm}$ de chuva. Por outro lado, observou-se que nos meses de junho, julho e agosto os valores de pluviosidade média mensal foram os menores, respectivamente 7, 26 e $20 \mathrm{~mm}$ de chuva (Tabela 2).

\section{Erosividade das chuvas}

A erosividade média mensal das chuvas variou de $7 \mathrm{MJ} \cdot \mathrm{mm} \cdot \mathrm{ha}^{-1} \cdot \mathrm{h}^{-1}$, no mês de junho, a 2.153 MJ.mm.ha-1 ${ }^{-1} \mathrm{~h}^{-1}$, no mês de janeiro (Tabela 3). O mês em que ocorreu a maior erosividade foi janeiro de 2007, totalizando $4.590 \mathrm{MJ} . \mathrm{mm} \cdot \mathrm{ha}^{-1} \cdot \mathrm{h}^{-1}$, resultado de $436 \mathrm{~mm}$ de chuva. No entanto, valores elevados de precipitação anual não necessariamente produzem maiores valores de erosividade. No Estado de Mato Grosso do Sul, a erosividade está associada a concentrações de chuvas em determinando período do ano, devido às características climáticas regionais (OLIVEIRA et al., 2012). Hoyos, Waylen e Jaramillo (2005) relatam que os elevados valores de erosividade observados em regiões tropicais são causados principalmente pela característica de formação das chuvas convectivas, resultando, assim, em valores elevados de total precipitado, intensidade e energia cinética da chuva.

Com exceção do mês de julho de 2008, nenhum mês da série apresentou zero de chuva (Tabela 2), apesar de vários meses apresentarem erosividade nula (Tabela 3 ). Isso se deve à ocorrência de chuvas não erosivas no período considerado.
A erosividade média anual das chuvas (Fator R) no período estudado foi de $10.440 \mathrm{MJ} \cdot \mathrm{mm} \cdot \mathrm{ha}^{-1} \cdot \mathrm{h}^{-1}$. Oliveira et al. (2012) verificaram que os valores de erosividade médios anuais para o Estado de Mato Grosso do Sul variam de 5.770 a $13.601 \mathrm{MJ} \cdot \mathrm{mm} \cdot \mathrm{ha}^{-1} \cdot \mathrm{h}^{-1}$. ano ${ }^{-1}$, com erosividade media anual de 9.318 $\pm 870 \mathrm{MJ} \cdot \mathrm{mm} \cdot \mathrm{ha}^{-1} \cdot \mathrm{h}^{-1} \cdot \mathrm{ano}^{-1}$. Dessa forma, pode-se verificar que o valor médio obtido no presente estudo é superior à média encontrada para o Estado. Além disso, pode-se classificar a erosividade média anual obtida como muito forte. A erosividade anual das chuvas apresentou variação, com valores entre 8.159 (forte) e 14.418 MJ.mm.ha ${ }^{-1} \cdot h^{-1}$ (muito forte), em 2008 e 2009, respectivamente. A determinação do Fator R possibilita a utilização da USLE no planejamento conservacionista do solo e da água.

\section{Coeficiente de chuva}

O coeficiente de determinação $\left(\mathrm{R}^{2}\right)$ de 0,97 , apresentado pela regressão potencial (Figura 1A), indica que $97 \%$ da variação dos valores observados foram explicados pelos valores calculados; consequentemente, $90 \%$ da variação dos valores observados foram explicados pela regressão linear (Figura 1B) e 65\% foram explicados pela equação de Lombardi Neto e Moldenhauer (1992) (Figura 1C).

Vários outros estudos também constataram que o índice pluviométrico possibilita obter resultados satisfatórios no cálculo da erosividade da chuva (RENARD \& FREIMUND, 1994; CARVALHO et al., 2005, HICKMANN et al., 2008; OLIVEIRA et al., 2012).

Os parâmetros estatísticos estimados para a regressão linear, potencial e de Lombardi Neto e Moldenhauer (1992) são apresentados na Tabela 4. As três equações apresentaram bom índice para coeficiente de Nash-Sutcliffe (COE). 
O coeficiente de massa residual (CMR) positivo para a equação de Lombardi Neto e Moldenhauer (1992) indica que essa equação tende a subestimar o índice de erosividade $\mathrm{EI}_{30}$, enquanto que os valores negativos para as regressões linear e potencial indicam que a erosividade é superestimada. Tal índice estatístico também confirma o melhor ajuste da regressão potencial, com desvios mais próximos de zero. O índice de eficiência (EF) apresentou-se aproximadamente igual às três equações, admitindo que não houve diferença entre elas. O coeficiente de ajuste (CA) foi melhor para a regressão potencial, ratificando essa equação como a mais adequada para estimar o índice de erosividade $\mathrm{EI}_{30}$ na área de estudo.

Os valores do Fator R da USLE estimados pelas equações linear e potencial, de 10.588 e $10.578 \mathrm{MJ} \cdot \mathrm{mm} \cdot \mathrm{ha}^{-1} \cdot \mathrm{h}^{-1} \cdot$ ano $^{-1}$, respectivamente,

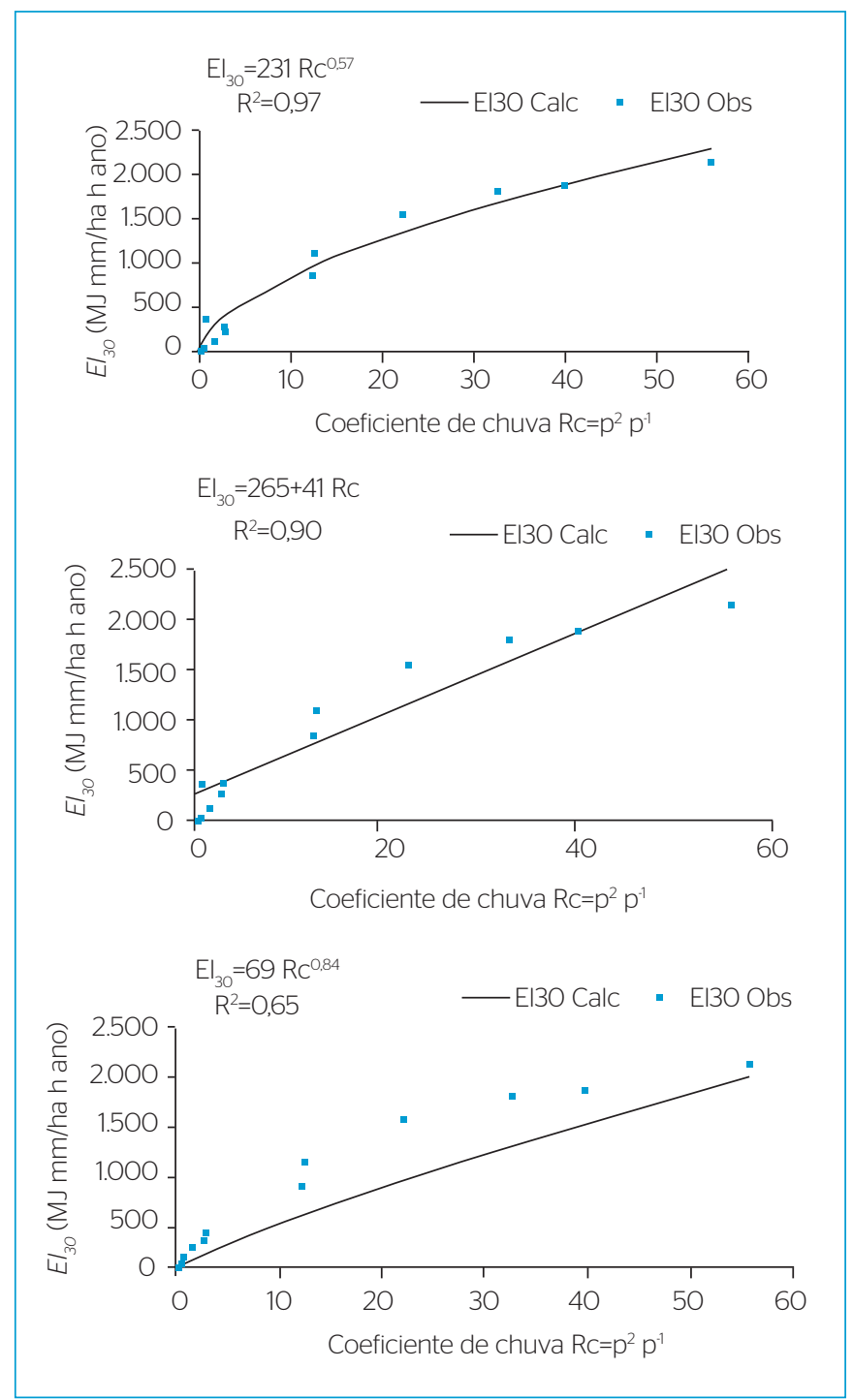

Figura 1 - Curvas de regressão potencial (A), linear (B) e de Lombardi Neto e Moldenhauer (1992) (C), entre o índice de erosividade $\mathrm{El}_{30}$ médio mensal e o coeficiente de chuva (Rc) para a bacia hidrográfica do Rio Taquarizinho (2006 a 2009). são próximos do valor observado de $10.440 \mathrm{MJ} \cdot \mathrm{mm} \cdot \mathrm{ha}^{-1} \cdot \mathrm{h}^{-1} \cdot \mathrm{ano}^{-1}$ (Tabela 5). Isso permite afirmar que o valor médio anual da erosividade das chuvas pode ser estimado por qualquer uma das equações obtidas por regressão, mesmo que a regressão potencial seja mais adequada. O valor de R de $7.354 \mathrm{MJ} \cdot \mathrm{mm} \cdot \mathrm{ha}^{-1} \cdot \mathrm{h}^{-1}$.ano ${ }^{-1}$, estimado pela equação Lombardi Neto e Moldenhauer (1992) para a região de estudo, subestimou em aproximadamente $30 \%$ os valores observados pelo índice $\mathrm{EI}_{30}$. Oliveira, Wendland e Nearing (2013) verificaram que as esquações de regressão para cálculo do Fator R não podem ser extrapoladas de forma generalizada, pois podem superestimar ou subestimar os valores de erosividade, como constatado no presente estudo. Os autores concluíram, ainda, que devem ser realizados estudos locais para aplicação dessas equações. Dessa forma, as equações obtidas no presente estudo podem auxiliar na aplicação da USLE para a região na qual foram desenvolvidas.

Devido à ausência de dados pluviográficos, Galdino, Padovani e Soriano (2004) obtiveram 7.348 MJ.mm.ha ${ }^{-1} \cdot \mathrm{h}^{-1}$.ano ${ }^{-1}$ para o Fator R na bacia do Rio Taquarizinho, com mais de 20 registros anuais completos de estações pluviométricas, utilizando a equação de Lombardi Neto (1977) desenvolvida para Campinas (SP). O valor encontrado foi semelhante ao deste estudo, utilizando a equação modificada (7354 MJ.mm.ha' ${ }^{-1} \cdot \mathrm{h}^{-1} \cdot$ ano $^{-1}$ ) entre os anos de 2006 e 2009, podendo-se perceber que a série de quatro anos observados demonstrou-se compatível com a série histórica estudada por aqueles autores.

Quanto à distribuição mensal dos valores de erosividade (Figura 2), de um modo geral, os valores estimados pelas regressões linear e potencial superestimaram os valores abaixo de $500 \mathrm{MJ} \cdot \mathrm{mm} \cdot \mathrm{ha}^{-1} \cdot \mathrm{h}^{-1} \mathrm{e}$ valores superiores a $2.000 \mathrm{MJ} \cdot \mathrm{mm} \cdot \mathrm{ha}^{-1} \cdot \mathrm{h}^{-1}$, enquanto a regressão potencial apresentou melhor ajuste para valores intermediários. A equação de Lombardi Neto e Moldenhauer (1992) apresentou o melhor ajuste para os valores abaixo de $300 \mathrm{MJ} \cdot \mathrm{mm} \cdot \mathrm{ha}^{-1} \cdot \mathrm{h}^{-1} \mathrm{e}$ valores superiores a $2.000 \mathrm{MJ} \cdot \mathrm{mm} \cdot \mathrm{ha}^{-1} \cdot \mathrm{h}^{-1}$.

Sugere-se, portanto, que a distribuição mensal da erosividade das chuvas seja estimada pelo uso da regressão potencial,

Tabela 4 - Parâmetros estatísticos estimados para a regressão linear, potencial e de Lombardi Neto e Moldenhauer (1992) para a bacia hidrográfica do Rio Taquarizinho (2006 e 2009).

\begin{tabular}{c|c|c|c} 
& Linear* & Potencial* $^{*}$ & Lombardi Neto e Moldenhauer (1992) \\
\hline COE & 0,90 & 0,96 & 0,80 \\
\hline CMR & $-0,01$ & $-0,01$ & 0,30 \\
\hline CA & 1,13 & 1,07 & 1,20 \\
\hline EF & $1,40.10^{-7}$ & $1,40 \times 10^{-7}$ & $1,30 \times 10^{-7}$ \\
\hline
\end{tabular}

COE: coeficiente de Nash-Sutcliffe; CMR: coeficiente de massa residual; CA: coeficiente de ajuste; EF: eficiência 
Tabela 5 - Regressão linear, potencial e de Lombardi Neto e Moldenhauer (1992) da quantidade de precipitação pluvial (mm) com a erosividade das chuvas (2006 a 2010).

\begin{tabular}{|c|c|c|c|c|c|c|}
\hline \multirow{2}{*}{ Mês do ano } & $\begin{array}{l}\text { Precipitação acumula- } \\
\text { da média mensal }\end{array}$ & $\begin{array}{l}\text { Coeficiente de chuva } \\
\text { (Rc) }\end{array}$ & $\begin{array}{l}\text { Indice de erosividade } \\
\qquad\left(\mathrm{El}_{30} \mathrm{Obs}\right)\end{array}$ & Regressão Linear & $\begin{array}{c}\text { Regressão Poten- } \\
\text { cial }\end{array}$ & $\begin{array}{l}\text { Lombardi Neto e } \\
\text { Moldenhauer (1992) }\end{array}$ \\
\hline & \multicolumn{2}{|c|}{$(\mathrm{mm})$} & \multicolumn{4}{|c|}{$\left(\mathrm{MJ} \mathrm{mm} \mathrm{ha} \mathrm{h}^{-1} \mathrm{~h}^{-1}\right)$} \\
\hline jan & 277 & 56 & 2.153 & 2.535 & 2.307 & 2.023 \\
\hline fev & 233 & 40 & 1.812 & 1.879 & 1.898 & 1.519 \\
\hline abr & 60 & 3 & 284 & 371 & 398 & 153 \\
\hline maio & 61 & 3 & 229 & 377 & 411 & 161 \\
\hline jun & 7 & 0 & 7 & 266 & 33 & 4 \\
\hline set & 43 & 1 & 123 & 321 & 275 & 89 \\
\hline out & 129 & 12 & 860 & 758 & 962 & 560 \\
\hline nov & 174 & 22 & 1.568 & 1.159 & 1.353 & 924 \\
\hline dez & 211 & 33 & 1.883 & 1.589 & 1.695 & 1.286 \\
\hline Total & 1.371 & 182 & 10.440 & 10.588 & 10.578 & 7.354 \\
\hline
\end{tabular}

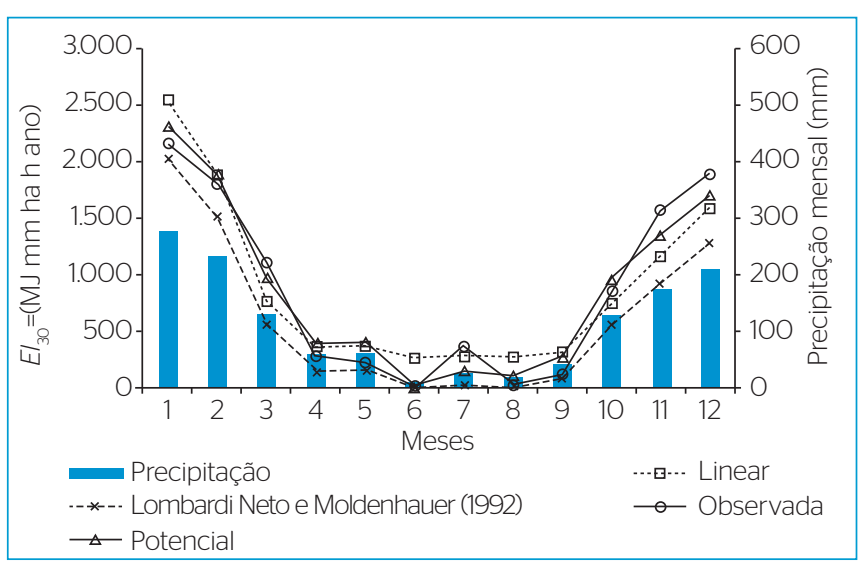

Figura 2 - Distribuição mensal da precipitação pluvial e erosividades observadas e calculadas pelas regressões linear, potencial e de Lombardi Neto e Moldenhauer (1992).

considerando os valores dos coeficientes estatísticos. A utilização da equação potencial dá consistência à relação obtida, uma vez que, se em determinado mês ocorrer zero de precipitação pluvial, a erosividade determinada será zero, fato que não se observará com a utilização da regressão linear, apresentando erosividade mínima de $265 \mathrm{MJ} \cdot \mathrm{mm} \cdot \mathrm{ha}^{-1} \cdot \mathrm{h}^{-1}$.

\section{CONCLUSÕES}

O valor médio observado para erosividade (10.440 MJ.mm.ha ${ }^{-1} \cdot \mathrm{h}^{-1}$. ano $^{-1}$ ) caracteriza elevado potencial erosivo das chuvas na bacia hidrográfica estudada. O período de outubro a março é o mais crítico em relação ao potencial erosivo, concentrando $90 \%$ da erosividade anual.

As equações de regressão linear e potencial determinadas no presente estudo apresentam melhores resultados que a equação de Lombardi Neto e Moldenhauer (1992). Portanto, deve-se evitar o uso de equações de regressão de outras regiões para o cálculo do Fator R da USLE, sem considerar as características climáticas da região estudada.

A equação de regressão potencial apresenta melhor resultado e consistência em relação aos valores observados; assim, sugere-se que tal equação seja usada para a estimativa da erosividade das chuvas para região estudada e de outras regiões de características climáticas semelhantes. 


\section{REFERÊNCIAS}

ANGULO-MARTÍNEZ, M. \& BEGUERÍA, S. (2009) Estimating rainfall erosivity from daily precipitation records among methods using data from the Ebro Basin (NE Spain). Journal of Hydrology, v. 379, p. 111-21.

ASSINE, M.L. (2005) River avulsions on the Taquari megafan, Pantanal wetland, Brazil. Geomorphology, v. 70, n. 3-4, p. 357-71.

CARVALHO, N.O. (2008) Hidrossedimentologia Prática. 2a ed. Rio de Janeiro: Interciência.

CARVALHO, D.F.; MONTEBELLER, C.A.; FRANCO, E.M.; VALCARCEL, R.; BERTOL, I. (2005) Padrões de precipitação e índices de erosividade para as chuvas de Seropédica e Nova Friburgo, RJ. Revista Brasileira de Engenharia Agrícola e Ambiental, v. 9, p. 7-14.

CASSOL, E.A.; ELTZ, F.L.F.; MARTINS, D.; LEMOS, A.M.; LIMA, V.S.; BUENO, A.C. (2008) Erosividade, padrões hidrológicos, período de retorno e probabilidade de ocorrência das chuvas em São Borja, RS. Revista Brasileira de Ciência do Solo, v. 32, p. 1239-51.

COLLINSCHONN, W; TUCCI, C.E.M; CLARKE, R.T. (2001) Further evidence of changes in the hydrological regime of the River Paraguay: part of a wider phenomenon of climate change? Journal of Hydrology, v. 245, n. $1-4$, p. $218-38$

FOSTER, G.R.; MCCOOL, D.K.; RENARD, K.G.; MOLDENHAUER, W.C. (1981) Conversion of the universal soil loss equation to SI units. Journal of Soil and Water Conservation, v. 36, p. 355-59.

GALDINO, S.L.; PADOVANI, C.R.; SORIANO, B.M.A. (2004) Erosão potencial laminar hídrica na Bacia do Alto Taquari. Revista Brasileira de Recursos Hídricos, v. 9, n. 2, p. 125-33.

HARRIS, M.B.; TOMAS, W.; MOURÃO, G.; SILVA, C.J.; GUIMARÃES, E.; SONODA, F; FACHIM, E. (2005) Safeguarding the Pantanal Wetlands: Threats and Conservation Initiatives. Conservation Biology, v. 19, p. 714-20.

HICKMANN C; ELTZ, F.L.F;; CASSOL, E.A.; COGO, C.M. (2008) Erosividade das chuvas em Uruguaiana, RS, determinada pelo índice El3O, com base no período de 1963 a 1991. Revista Brasileira de Ciência do Solo, v. 32, p. 825-31.

HOYOS, N.; WAYLEN, P.R.; JARAMILLO, A. (2005) Seasonal and spatial patterns of erosivity in a tropical watershed of the Colombian Andes. Journal of Hydrology, v. 314, p. 177-91.

INSTITUTO BRASILEIRO DE GEOGRAFIA E ESTAÍSTICA - IBGE (2010). Disponível em: <http://www.ibge.gov.br>. Acesso em: 17 ago. 2010.

JUNK, W.J. \& CUNHA, C.N. (2005) Pantanal: a large South American wetland at a crossroads. Ecological Engineering, v. 24, n. 4, p. 391-01.

LOMBARDI NETO, F. \& MOLDENHAUER, W.C. (1992) Erosividade da chuva: sua distribuição e relação com perdas de solo em Campinas, SP. Bragantia, v. 51, p. 189-96.

LOMBARDI NETO, F. (1977) Rainfall erosivity - Its distribution and relationship with soil loss at Campinas, Brazil. MSc Thesis, Purdue University, West Lafayette.
MEUSBURGER, K.; STEEL, A.; PANAGOS, P.; MONTANARELLA, L; ALEWELL, C. (2012) Spatial and temporal variability of rainfall erosivity factor for Switzerland. Hydrology and Earth System Sciences, v. 16, p. 167-77.

MIKOS, M.; JOST, D.; PETKOVSEK, G. (2006) Rainfall and runoff erosivity in the alpine climate of north Slovenia: a comparison of different estimation methods. Hydrological Sciences, v. 51, n. 1, p. 115-26.

OLIVEIRA, P.T.S.; ALVES SOBRINHO, T. RODRIGUES, D.B.B.; PANACHUKI, E. (2011) Erosion Risk Mapping Applied to Environmental Zoning. Water Resources Management, v. 25, p. 1021-36.

OLIVEIRA, P.T.S.; RODRIGUES, D.B.B.; ALVES SOBRINHO, T.; CARVALHO, D.F.; PANACHUKI, E. (2012) Spatial variability of the rainfall erosive potential in the State of Mato Grosso do Sul, Brazil. Engenharia Agrícola, v. 32, p. 69-79.

OLIVEIRA, P.T.S., WENDLAND, E., NEARING, M.A. (2013) Rainfall erosivity in Brazil: A Review. Catena, v. 100, p. 139-47.

PAZ, A.R.; COLLISCHONN, W.; TUCCI, C.E.M.; PADOVANI, C.R. (2O11) Large-scale modelling of channel flow and floodplain inundation dynamics and its application to the Pantanal (Brazil). Hydrological Processes, v. 25, n. 9, p. 1498-16.

REBOUÇAS, A.C.; BRAGA, B.; TUNDISI, J.G. (2002) Águas doces no Brasil: capital ecológico, uso e conservação. 2 ed. São Paulo: Escrituras Editora.

RENARD, K.G.; FOSTER, G.R.; WEESIES, G.A. (1997) Predicting soil erosion by water: a guide to conservation planning with the revised universal soil loss equation (RUSLE). Agriculture Handbook, n. 703, USDA-ARS.

RENARD, K.G.; FREIMUND, J.R. (1994) Using monthly precipitation data to estimate the R-factor in the revised USLE. Journal of Hydrology, v. 157, p. 287-06.

SALLES, C. \& POESEN, J. (2000) Rain properties controlling soil splash detachment. Hydrological Processes, v. 14, p. 271-82

SCHAEFLI, B. \& GUPTA, H. (2007) Do Nash values have value? Hydrological Processes, v. 21, p. 2075-80.

SILVA, A.M.; WIECHETECK, M.; ZUERCHER, B. (2011) Spatial assessment of indices for characterizing the erosive force of rainfall in El Salvador Republic. Environmental Engineering Science, v. 28, n. 4, p. 309-316.

SILVA, A.M. (2004) Rainfall erosivity map for Brazil. Catena, v. 57, p. 251-59.

SORIANO, B.M.A.; GALDINO, S.; CLARKE, R.T. (2001) Evolução da erosividade das chuvas na Bacia do Alto Taquari. Corumbá: Embrapa Pantanal. Boletim de Pesquisa, n. 25, 18 p.

WISCHMEIER, W.H. (1959) A rainfall erosion index for a universal-loss equation. Soil Science Society of America, v. 23, p. 246-49.

YU, B.; HASHIM, G.M.; EUSOF, Z. (2001) Estimating the R-factor with limited rainfall data: A case study from Peninsular Malaysia. Journal of Soil and Water Conservation, v. 56, n. 2, p. 101-5. 


\section{Errata}

Revista Engenharia Sanitária e Ambiental 2014;19(2): 195

DOI: $10.1590 /$ S1413-41522014000200010

Artigo: Erosividade da chuva para o bioma Pantanal

Na página 195, na titulação da autora Aline da Silva Ribeiro, onde se lê:

Engenheira Ambiental pela UFMS. Mestre e Doutoranda em Hidráulica e Saneamento pela Escola de Engenharia de São Carlos da Universidade de São Paulo (EESC/USP) - São Carlos (SP), Brasil.

Leia-se:

Engenheira Ambiental pela UFMS. Mestre em Hidráulica e Saneamento pela Escola de Engenharia de São Carlos da Universidade de São Paulo (EESC/USP). Doutoranda em Engenharia Hidráulica Sanitária pela Escola Politécnica da Universidade de São Paulo (POLI/USP). Pesquisadora do Instituto de Pesquisas Tecnológicas (IPT/SP) - São Paulo (SP), Brasil. 